\section{Apremilast stoppt \\ Psoriasis-bedingten Juckreiz}

Der Phosphodiesterase-4-Hemmer Apremilast, der unter anderem zur Therapie der mittelschweren bis schweren Plaque-Psoriasis zugelassen ist, lindert offenbar auch den krankheitsbedingten Juckreiz und verhilft den Betroffenen zu einem besseren Hautgefühl.

A nhand der Daten zweier randomisierter placebokontrollierter Phase-3-Studien (ESTEEM 1 und ESTEEM 2), in denen die Wirksamkeit und Sicherheit des Wirkstoffs Apremilast bei moderater bis schwerer Plaque-Psoriasis untersucht worden waren, haben US-Dermatologen überprüft, inwieweit der Wirkstoff auch den häufig auftretenden Juckreiz und die Missempfindungen lindern kann. Von den 1.255 Probanden der beiden industriegesponserten Untersuchungen hatten 836 zweimal täglich 30 mg Apremilast eingenommen, 419 Placebo. Alle Patienten wurden zwei, vier und acht Wochen sowie anschließend über sechs Monate hinweg alle vier Wochen nachuntersucht. Zwei Drittel der Teilnehmer litten zu Studienbeginn an einer moderaten Plaque-Psoriasis, etwa ein Drittel war schwer erkrankt.

Bereits nach zwei Wochen Therapie hatte sich in beiden Studien im Vergleich zu Placebo eine signifikante Verbesserung in puncto Juckreiz und Missempfindungen eingestellt $(\mathrm{p}<0,0001)$. Nach 16 Wochen waren die Scores auf der visuellen Analogskala um 47,6\% (ESTEEM 1) und 49,4\% (ESTEEM 2) gesunken. Hatten die Probanden anfangs ihren Juckreiz bei $66 \mathrm{~mm}$ (ESTEEM 1) und $68 \mathrm{~mm}$ (ESTEEM 2) auf der VAS eingestuft, gaben sie 16 Wochen später die Intensität nur noch mit $35 \mathrm{~mm}$ und 34 $\mathrm{mm}$ an. Der therapeutische Effekt war auch 32 Wochen später noch nachweisbar. Ein ähnliches Ergebnis ergab sich auch bei der Beurteilung der Psoriasis-bedingten Missempfindungen oder Schmerzen (VAS). Die Schmerzintensität hatte sich nach 16 Wochen Therapie um etwa $50 \%$ verringert.

Die Daten zeigten zudem eine Korrelation zwischen Rückgang des Pruritus und Verbesserung des Dermatologischen Lebensqualitätsindex-Scores (in beiden Studien $\mathrm{p}<0,001$ ) sowie zwischen Krankheitsaktivität (PgAPDA) und Intensität von Pruritus (je $\mathrm{p}<0,001$ ) und Missempfindungen (je $\mathrm{p}<0,001$ ).

Fazit: Bei Patienten mit Plaque-Psoriasis verbessern sich unter dem Wirkstoff Apremilast die Symptome Juckreiz und Missempfindungen signifikant. Meist war nach 16 Wochen ein klinisch relevanter Effekt nachweisbar, wobei die meisten Patienten bereits nach zwei Wochen eine erste Besserung verspürten. Die Dermatologen bedauern, dass in den meisten Scores zur Bestimmung der Krankheitsaktivität (z.B. PASI) die Symptome Juckreiz und Missempfindung nicht berücksichtigt werden, und das obwohl so viele Patienten darunter leiden und sich dadurch in ihrer Lebensqualität eingeschränkt fühlen. Dr. Dagmar Kraus

Sobell JM et al. Effects of Apremilast on Pruritus and Skin Discomfort/Pain Correlate With Improvements in Quality of Life in Patients With Moderate to Severe Plaque Psoriasis. Acta Derm Venereol 2016; 96: 514-20

\section{Hier steht eine Anzeige.}

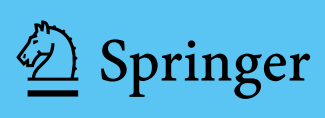

